\title{
Fission yield covariances for JEFF: A Bayesian Monte Carlo method
}

\author{
Olivier Leray ${ }^{1, \text { a }}$, Dimitri Rochman ${ }^{1}$, Michael Fleming ${ }^{2}$, Jean-Christophe Sublet ${ }^{2}$, Arjan Koning ${ }^{3,4}$, Alexander Vasiliev ${ }^{1}$, \\ and Hakim Ferroukhi ${ }^{1}$ \\ ${ }^{1}$ Paul Scherrer Institut, 5232 Villigen, Switzerland \\ 2 United Kingdom Atomic Energy Authority, Abingdon, UK \\ 3 Nuclear Data Section, IAEA, Vienna, Austria \\ 4 University of Uppsala, Sweden
}

\begin{abstract}
The JEFF library does not contain fission yield covariances, but simply best estimates and uncertainties. This situation is not unique as all libraries are facing this deficiency, firstly due to the lack of a defined format. An alternative approach is to provide a set of random fission yields, themselves reflecting covariance information. In this work, these random files are obtained combining the information from the JEFF library (fission yields and uncertainties) and the theoretical knowledge from the GEF code. Examples of this method are presented for the main actinides together with their impacts on simple burn-up and decay heat calculations.
\end{abstract}

\section{Introduction}

The Bayesian Monte-Carlo (BMC) method applied to fission yields has been introduced in [1]. This method combines theoretical fission yields with a set of reference data. In this study the GEF code [2,3] provides the theoretical fission yields and uncertainties which are fitted to the JEFF-3.1.1 data considered as the reference. The advantage of this process is to provide fission yield correlations to an existing nuclear data evaluation. Two applications of uncertainty propagation using these correlated fission yields are presented in the following. First a PWR fuel assembly depletion case is simulated and both the uncertainties on the k-inf and on the nuclide composition are shown. Finally benchmarks using the above-mentioned fission yields and assessing the decay heat uncertainty are presented.

\section{The Bayesian Monte Carlo approach}

\subsection{Principle of the method}

The GEF code provides yields for spontaneous fission and particle-induced fission and for a very large range of excitation energy of the nucleus (up to $100 \mathrm{MeV}$ ). GEF is based on physical models and its fission yields are in good agreement with recent evaluations and experimental data. About 50 parameters are adjusted in GEF to fit the systems simulated. In this analysis only the 21 most important are independently and randomly (following a Gaussian law) sampled to create a set of perturbed fission yields. The classical stochastic method is improved in this case by a Bayesian feedback. A $\chi^{2}$ (Eq. (1)), taking into account the fission yield uncertainty $\sigma$, is computed for every set of perturbed fission yield $\mathrm{FY}_{\mathrm{CAL}}$ assessing the

a e-mail: olivier.leray@psi.ch discrepancy between the latter and a reference value $F Y_{\text {REF }}$ (for instance from an international evaluation).

$$
\chi^{2}=\sum_{i=1}^{F Y}\left(\frac{F Y_{c a l_{i}}-F Y_{R E F_{i}}}{\sigma_{F Y_{R E F_{i}}}}\right)^{2}
$$

Then a weight (Eq. (2)) based on the $\chi^{2}$ is associated to every perturbed set of GEF parameters $j$.

$$
\omega_{j}=e^{-\frac{\chi_{j}^{2}}{2}} / e-\frac{\chi_{\min }^{2}}{2}
$$

The distributions of the GEF parameters are in this way updated, their mean value and/or standard deviation can change and they may not be Gaussian anymore. Nevertheless, only the two first moments (mean and standard deviation) are updated and re-used for the next iteration as parameter of the Gaussian they are approximated (there is no update of the distribution). From this point, new perturbed fission yields are produced and the iterative process is repeated until convergence. More details on the method, weights and $\chi^{2}$ are exposed in [1].

\subsection{Application}

This method was applied to the JEFF-3.1.1 independent fission yields (taking the mean value and standard deviation as reference) for 4 different systems: the thermal fission (at $25.3 \mathrm{meV}$ ) of ${ }^{235} \mathrm{U},{ }^{239} \mathrm{Pu}$ and ${ }^{241} \mathrm{Pu}$ and fast fission (at $500 \mathrm{keV}$ ) of ${ }^{238} \mathrm{U} .11$ iterations were performed and several hundred of perturbed independent (MT454) and cumulative (MT459) fission yields were generated by GEF using these fitted parameters. For computational purposes, only 500 sampled sets are used to the different applications below.

(c) The Authors, published by EDP Sciences. This is an Open Access article distributed under the terms of the Creative Commons Attribution License 4.0 (http://creativecommons.org/licenses/by/4.0/). 


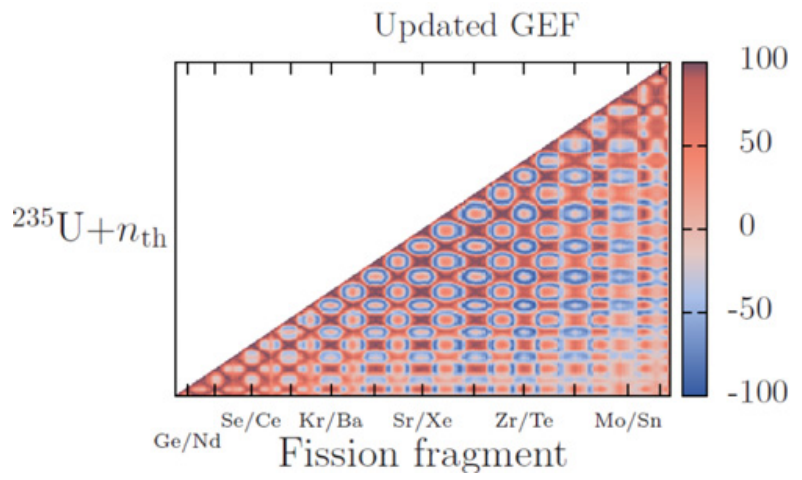

Figure 1. Correlations between the fission yields from ${ }^{235} \mathrm{U}$ (thermal fission).

The same seed for the stochastic sampling of the different fissioning systems is used. For instance, the same seed is used to sample the ${ }^{235} \mathrm{U}+\mathrm{n}_{t h}$ and the ${ }^{239} \mathrm{Pu}+\mathrm{n}_{t h}$ yields. As a result, the fission yields from one system are not only correlated between them but also with the other yields of other fissioning nuclei $\left(e . g\right.$. between ${ }^{235} \mathrm{U}+\mathrm{n}_{t h}$ and $\left.{ }^{239} \mathrm{Pu}+\mathrm{n}_{t h}\right)$. Those "cross-correlations", due solely to the model, are kept and propagated in the simulations of the next section.

\section{Impacts on lattice $\mathbf{k}_{\text {inf }}$, nuclide density and decay heat}

The Shark-X [4] tool at PSI is based on a modified version of the 2D deterministic neutron physics code CASMO-5. It allows taking into account external files containing perturbation factors for the nuclear data. Hence Shark-X is used for Uncertainty Quantification (UQ) on nuclear data studies. Among other nuclear data, fission yields can be perturbed according to different methods to introduce correlations (or not) between the yields. One capability of this tool is to read directly fission yields (independent and cumulative) from ENDF-6-formatted files and adapt them to the CASMO-5 limited list of nuclides. If a set of perturbed fission yields in ENDF files is provided, the perturbation factors can be generated and be used for a Monte Carlo propagation through CASMO-5. Finally the UQ of the output parameters is performed by a statistical analysis of the perturbed output files. One important point to keep in mind is that the average value of the perturbed sample of fission yields in CASMO-5 is not changed and remains for all calculation the mean value provided by ENDF/B-VII.1. Only different uncertainty sources are applied. The system chosen for this analysis is a PWR assembly constituted by a 15 by $15 \mathrm{UO}_{2}$ fuel pins lattice. The analysis is performed at hot full power conditions with $900 \mathrm{ppm}$ boron concentration. 4 gadolinium pins $(2 \mathrm{w} \%$ of Gadolinium and $4.12 \%$ enrichment in $\left.{ }^{235} \mathrm{U}\right), 16$ guide tubes and one instrumentation pin are dispatched among the 204 fuel pins $\left(4.12 \%{ }^{235} \mathrm{U}\right.$ enriched) as shown in Fig. 2. The depletion calculation is performed up to $60 \mathrm{GWd} / \mathrm{t}$ with a power density of $33.75 \mathrm{~W} / \mathrm{gU}$.

The complete specifications for this fuel assembly can be found in [5]. This case was also chosen to ease the comparison with other sources of fission yield uncertainties which are already quantified on the same system [6].

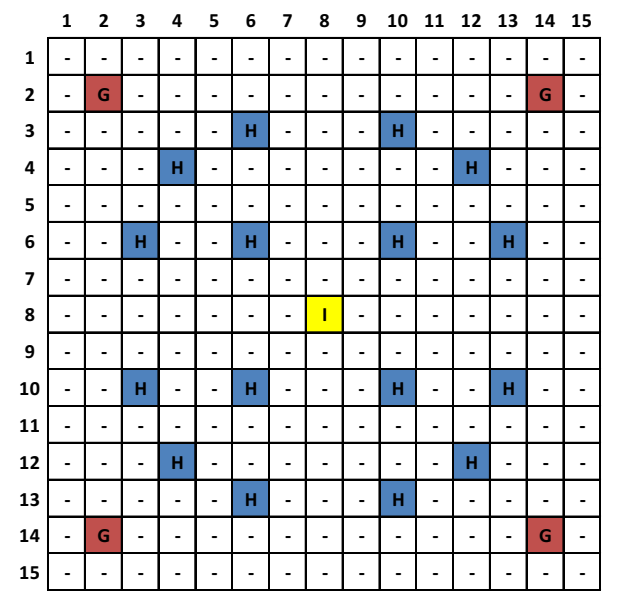

Figure 2. Fuel assembly lattice layout. Gadolinium pins, guide tubes and the instrumentation rod are located respectively at the $\mathrm{G}, \mathrm{H}$ and I marks.

Table 1. Nuclide density uncertainty (\%) at $60 \mathrm{GWd} / \mathrm{t}$ burnup.

\begin{tabular}{|l|l|l|l|l|l|}
\hline${ }^{90} \mathrm{Sr}$ & 4.53 & ${ }^{137} \mathrm{Cs}$ & 2.92 & ${ }^{151} \mathrm{Sm}$ & 15.12 \\
\hline${ }^{95} \mathrm{Mo}$ & 3.00 & ${ }^{144} \mathrm{Ce}$ & 4.94 & ${ }^{152} \mathrm{Sm}$ & 18.25 \\
\hline${ }^{99} \mathrm{Tc}$ & 2.77 & ${ }^{142} \mathrm{Nd}$ & 3.44 & ${ }^{153} \mathrm{Eu}$ & 19.09 \\
\hline${ }^{101} \mathrm{Ru}$ & 2.61 & ${ }^{143} \mathrm{Nd}$ & 3.63 & ${ }^{154} \mathrm{Eu}$ & 19.70 \\
\hline${ }^{103} \mathrm{Rh}$ & 7.33 & ${ }^{144} \mathrm{Nd}$ & 4.21 & ${ }^{155} \mathrm{Eu}$ & 21.70 \\
\hline${ }^{109} \mathrm{Ag}$ & 13.15 & ${ }^{145} \mathrm{Nd}$ & 5.45 & ${ }^{155} \mathrm{Gd}$ & 22.49 \\
\hline${ }^{129} \mathrm{I}$ & 14.16 & ${ }^{146} \mathrm{Nd}$ & 6.15 & ${ }^{156} \mathrm{Gd}$ & 25.74 \\
\hline${ }^{131} \mathrm{Xe}$ & 7.36 & ${ }^{148} \mathrm{Nd}$ & 9.80 & ${ }^{157} \mathrm{Gd}$ & 36.45 \\
\hline${ }^{135} \mathrm{Xe}$ & 2.67 & ${ }^{147} \mathrm{Sm}$ & 7.73 & ${ }^{158} \mathrm{Gd}$ & 49.46 \\
\hline${ }^{133} \mathrm{Cs}$ & 4.21 & ${ }^{149} \mathrm{Sm}$ & 11.29 & & \\
\hline${ }^{134} \mathrm{Cs}$ & 3.90 & ${ }^{150} \mathrm{Sm}$ & 10.79 & & \\
\hline
\end{tabular}

\subsection{Fission yield uncertainty on $\mathbf{k}_{\text {inf }}$}

The impact on k-inf of the fission yields uncertainties from ${ }^{235,238} \mathrm{U}$ and ${ }^{239,241} \mathrm{Pu}$ produced by the Bayesian process explained in Sect. 2 are presented in Fig. 3. At low burnup ${ }^{235} \mathrm{U}$ is the main contributor to the uncertainty while it is ${ }^{239} \mathrm{Pu}$ at higher burnup. The total uncertainty propagated by the perturbation of all fission yields ("ALL" curve) reaches $0.50 \%$ uncertainty and is much larger than the quadratic sum of the separated uncertainties ("SUM" curve). This indicates the strong effect of the cross-correlations (e.g., between ${ }^{235} \mathrm{U}$ and ${ }^{239} \mathrm{Pu}$ ) on the k-inf.

Finally the uncertainty on k-inf due to the fission yields using this method could be compared to those in [6]. The JEFF-3.1.1 uncertainties result in higher k-inf uncertainty compared to ENDF/B-VII.1 uncertainties.

\subsection{Nuclide density}

Uncertainty on nuclide composition of spent fuel is valuable for many application such as radiotoxicity, decay heat, transport or burnup credit. The uncertainty on the main isotopes produced during the depletion is retrieved by computing the standard deviation of the nuclide densities in all perturbed calculations (at end of irradiation, i.e., at $60 \mathrm{GWd} / \mathrm{t}$ ). Table 1 presents the uncertainties for a selection of nuclide compositions.

Some uncertainties are quite large compared to other evaluation (ENDF/B-VII.1) or method (SANDY) [6], especially when considering the Europium, Samarium and 


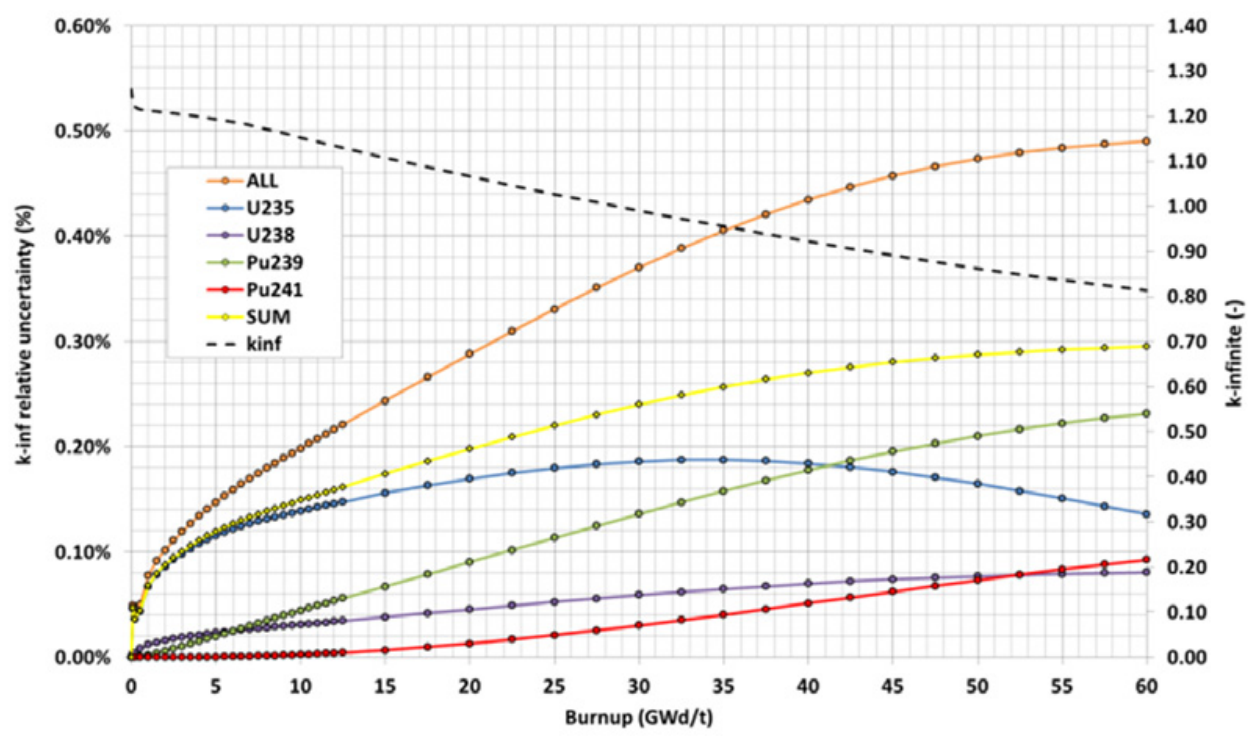

Figure 3. k-inf relative uncertainty due to fission yields (left axis) and k-inf (right axis).

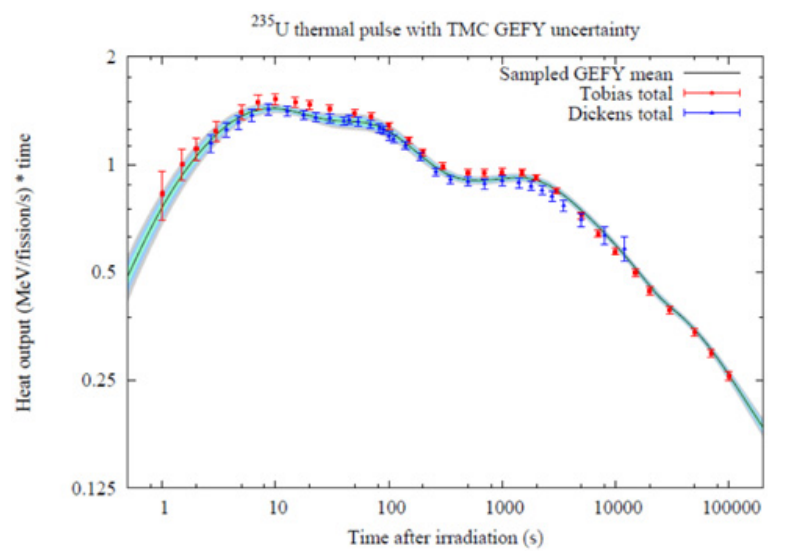

Figure 4. Decay heat from a thermal pulse on ${ }^{235} \mathrm{U}$ calculated with FISPACT-II using the 500 sampled fission yields.

Gadolinium isotopes which have more than $10 \%$ uncertainty. One can note also a large uncertainty on the ${ }^{148} \mathrm{Nd}$. This isotope composition is calculated in CASMO-5 by the mean of the cumulative fission yield which is in our case not fitted. To fit both the independent and cumulative fission yields is a way of improvement for the method.

\subsection{Decay heat benchmarks}

The fission yields have a strong impact on the decay heat and are the main source of uncertainties [7]. A reliable assessment of the latter becomes difficult as no correlations between the fission yields are provided in the nuclear data evaluations. In the present case, the propagation of the uncertainty and their correlations are taken into account by using the perturbed fission yields stored in the ENDF-6formatted files with the FISPACT-II code [8]. It simulates the beta and gamma emissions after a short pulse of thermal neutron on a target $\left({ }^{235} \mathrm{U}\right.$ in Fig. 4). The mean and standard deviation of the decay heat is then computed over the 500 calculations. The uncertainties are generally lower than the experimental data (Tobias [9] ,Dickens [10]).

From Fig. 5 it can be seen also that the JEFF-3.1.1 uncertainties are larger on decay heat than those from

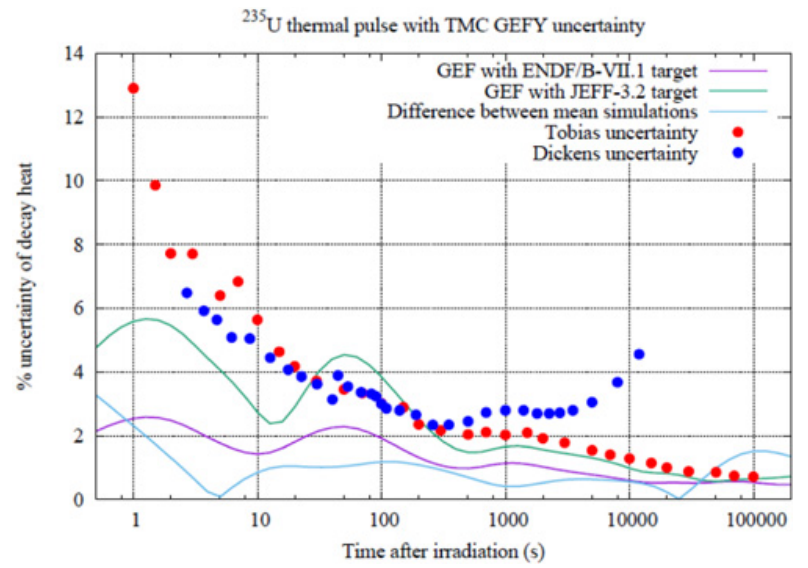

Figure 5. Decay heat uncertainty from a thermal pulse on ${ }^{235} \mathrm{U}$. Comparison of the JEFF-3.1.1 and ENDF/B-VII.1 uncertainties.

ENDF/B-VII.1 using the same Bayesian Monte-Carlo process (work presented in [1]).

\section{Conclusion}

A Bayesian approach has been applied to the uncertainty propagation of fission yields provided by the GEF code. An iterative process is used to fit the GEF independent fission yields to those of JEFF-3.1.1. This method produces correlations between the fission yields. The application of these new sets of fission yields to a simple burn-up case shows an uncertainty on the $\mathrm{k}$-inf below $0.50 \%$ at high burnup which is increased by the correlations introduced between the fission yields of different fissioning systems. The resulting uncertainty on the nuclide compositions at $60 \mathrm{GWd} / \mathrm{t}$ is in some cases (Sm, Eu and Gd isotopes) larger than 10\% and highlight a limitation of the method since the cumulative fission yields are not fitted. Also the use of ENDF/B-VII.1 fission yields with JEFF-3.1.1 uncertainties may be a cause of these large uncertainties which are not observed in the case of the decay heat benchmark where consistent mean values and uncertainties (all from JEFF-3.1.1) are employed in 
the calculations. The uncertainty on the decay heat due to thermal neutron pulses is shown to be lower than the experimental uncertainty. Finally on the Bayesian Monte-Carlo method, the mean chi square may not be a reliable criterion to check the convergence, the complete chi square distribution should be analysed instead.

\section{References}

[1] D. Rochman, O. Leray, A. Vasiliev, H. Ferroukhi, A.J. Koning, M. Fleming and J.-C. Sublet, "A Bayesian Monte Carlo method for fission yield covariance information," Annals of Nuclear Energy 95, 125-134 (2016)

[2] K.-H. Schmidt, B. Jurado, C. Amouroux and C. Schmitt, "General description of fission observables: GEF model code," Nuclear Data Sheets 131, 107-221 (2016)

[3] K. Schmidt and B. Jurado, "GEF A General Description of Fission Observables," OECD Nuclear Energy Agency, JEFF report 24, NEA/DB/DOC(2014)1

[4] W. Wieselquist, T. Zhu, A. Vasiliev and H. Ferroukhi, "PSI Methodologies for Nuclear Data Uncertainty Propagation with CASMO-5M and MCNPX: Results for OECD/NEA UAM Benchmark Phase I," Science and Technology of Nuclear Installations 2013 Article ID 549793 (2013)

[5] T. Blyth, N. Porter, M. Avramova, K. Ivanov, E. Royer, E. Sartori, O. Cabellos, H. Ferroukhi and
E. Ivanov, "Benchmark for Uncertainty Analysis in Modelling (UAM) for Design, Operation and Safety Analysis of LWRs, Volume II: Specifications and Support Data for the Core Cases (Phase II)," NEA/NSC/DOC(2014), Version 2.0, OECD Nuclear Energy Agency, April, 2014

[6] O. Leray, L. Fiorito, D. Rochman, H. Ferroukhi, A. Pautz, A. Stankovskiy and G. Van den Eynde, "Comparison of Fission Yield Perturbation Methodologies on Nuclide Composition for a PWR UO2 Fuel Assembly," in Proc. of the Int. Conf. PHYSOR2016, Sun Valley, Idaho, USA, 2016

[7] H. Ferroukhi, O. Leray, M. Hursin, A. Vasiliev, G. Perret and A. Pautz, "Study of Nuclear Decay Data Contribution to Uncertainties in Heat Load Estimations for Spent Fuel Pools," Nuclear Data Sheets 118, 498-501 (2014)

[8] J.-C. Sublet, J. Eastwood, J. Morgan, M. Fleming and M. Gilbert, "The FISPACT-II User Manual," UKAEA-R(11) 11 Issue 7, UKAEA, October 2015

[9] A. Tobias, "Derivation of Decay Heat benchmarks for ${ }^{235} \mathrm{U}$ and ${ }^{239} \mathrm{Pu}$ by a least squares fit to a measured data," Central Electricity Generating board, p. CEGB RD/B/6210/R89, 1989

[10] J. Dickens and et al., "Delayed beta and gamma-ray production due to thermal neutron fission of ${ }^{235} \mathrm{U}$ : Spectral distributions for times after fission between 2 and 14000s," ORNL/NUREG-39 (NUREG/CR0162), Oak Ridge National Laboratory, 1978 